\title{
Entropy scaling and simulability by Matrix Product States
}

\author{
Norbert Schuch, ${ }^{1}$ Michael M. Wolf, ${ }^{1}$ Frank Verstraete, ${ }^{2}$ and J. Ignacio Cirac ${ }^{1}$ \\ ${ }^{1}$ Max-Planck-Institut für Quantenoptik, Hans-Kopfermann-Str. 1, D-85748 Garching, Germany. \\ ${ }^{2}$ Fakultät für Physik, Universität Wien, Boltzmanngasse 5, A-1090 Wien, Austria.
}

\begin{abstract}
We investigate the relation between the scaling of block entropies and the efficient simulability by Matrix Product States (MPS), and clarify the connection both for von Neumann and Rényi entropies, as summarized in Table [. Most notably, even states obeying a strict area law for the von Neumann entropy are not necessarily approximable by MPS. We apply these results to illustrate that quantum computers might outperform classical computers in simulating the time evolution of quantum systems, even for completely translational invariant systems subject to a time independent Hamiltonian.
\end{abstract}

PACS numbers: 03.67.Mn, 03.65.Ud, 03.67.Lx, 05.10.Cc

Understanding the behaviour of quantum many-body systems is a central problem in physics. Recently, Matrix Product States (MPS) have received much interest as a variational ansatz for the simulation of correlated one-dimensional systems. They have proven particularly powerful in approximating the ground states of local Hamiltonians, as used in the DMRG method [1, 2], but have also been applied, e.g., to simulate the time evolution of slightly entangled quantum systems [3]. Despite considerable progress [4], it is still not fully understood which property exactly a state has to fulfil to be well approximated by MPS. This knowledge is not only of practical interest, but could also tell us how to extend the MPS ansatz to, e.g., higher dimensional systems.

It is generally believed that the relevant criterion for efficient approximability by MPS is that the states under consideration obey an area law, i.e., the von Neumann entropy of a block is bounded. Although indeed both ground states of local Hamiltonians and MPS obey an area law, there are reasons to doubt this immediate connection: Firstly, the von Neumann entropy is an asymptotic concept, quantifying what happens when dealing with a large number of copies of a state. Conversely, it has been shown recently that a rigorous connection can be established by looking at Rényi entropies instead [5]. Unfortunately, the argument used breaks down as the von Neumann entropy is approached. Finally, the conti-

\begin{tabular}{|c|c|c|c|c|}
\hline$S_{\alpha^{\sim}}$ & const & $\log L$ & $L^{\mathrm{K}}(\mathrm{K}<1)$ & $L$ \\
\hline$S_{\alpha<1}$ & \multicolumn{2}{|c|}{ approximable } & \multicolumn{2}{|c|}{$i n e^{d}$} \\
\hline$S \equiv S_{1}$ & \multirow{2}{*}{\multicolumn{2}{|c|}{$u n d e^{t e}$}} & & \\
\hline$S_{\alpha>1}$ & & & \multicolumn{2}{|c|}{ inapproximable } \\
\hline
\end{tabular}

TABLE I: Relation between scaling of block Rényi entropies and approximability by MPS. In the "undetermined" region, nothing can be said about approximability just from looking at the scaling. nuity inequality for the von Neumann entropy carries a size-dependent constant, and thus states which are close to each other need not be close in entropy [ [6].

In this work, we explore the connection between entropy scaling and approximability by MPS. The results are summarized in Table If An at most logarithmic scaling of Rényi entropies $S_{\alpha}, \alpha<1$, implies approximability by MPS. On the other side, a faster than logarithmic increase of $S_{\alpha}, \alpha>1$, rules out efficient approximability by MPS, as does linear growth of the von Neumann entropy. For all other cases, the scaling of the block entropy does not allow for conclusions about approximability. In particular, this holds for the case of constant von Neumann entropy, which demonstates that the reason why MPS describe ground states well is not simply that those states obey an area law.

Finally, we apply our results to illustrate that quantum computers might outperform classical computers in simulating time evolutions. It is long-known that quantum computers can simulate the behavior of quantum systems [7]. However, this does not automatically imply that they will outperform classical computers, as, e.g., ground states of gapped quantum systems appear classically efficiently approximable [4, 8]. On the other hand, it is known that time evolution even of one-dimensional systems under a translational invariant Hamiltonian can implement quantum computations if either translational invariance is broken by the initial, boundary, or final conditions, or the Hamiltonian is time dependent [9], and is thus hard to simulate. We extend these results by showing that even the simplest case, the evolution of a translational invariant spin $\frac{1}{2}$ system with translational invariant initial conditions under a time independent Hamiltonian, cannot be simulated efficiently using MPS; this provides evidence that quantum computers might outperform classical computers in simulating these systems.

Let us first introduce the relevant quantities and notations. We want to obtain approximations which reproduce accurately not only the local properties such as energy, but also the non-local ones such as correlations. This is ensured by bounding the error made between two 
states $|\psi\rangle$ and $|\phi\rangle$ for an arbitrary observable $O$,

$$
|\operatorname{tr}[\psi O]-\operatorname{tr}[\phi O]| \leq\|O\|_{\text {op }}\|\psi-\phi\|_{\text {tr }},
$$

where throughout the paper, $\psi \equiv|\psi\rangle\langle\psi|$ etc. denotes the corresponding density operator. We focus on nonextensive observables (see Footnote [17] for extensive observables), therefore w.l.o.g. $\|O\|_{\text {op }} \leq 1$. It follows that by imposing

$$
\|\psi-\phi\|_{\mathrm{tr}} \leq \delta,
$$

we bound the error made in any observable by $\delta$.

For some of the proofs it will be more convenient to consider the two-norm distance $\||\psi\rangle-|\phi\rangle \|_{2}$ or the fidelity $|\langle\phi \mid \psi\rangle| / \||\phi\rangle\left\|_{2}\right\||\psi\rangle \|_{2}=: \cos (\theta)$. Fortunately, these measures turn all out to be equivalent: Since the best approximating MPS will generally not be normalized, it is appropriate to consider the optimized quantities, and one finds that $T(\phi, \psi):=\inf _{\alpha}\|\psi-\alpha \phi\|_{\text {tr }} /\|\psi\|_{\text {tr }} \equiv \sin (2 \theta)$ and $V(\phi, \psi):=\inf _{\alpha} \||\psi\rangle-\alpha|\phi\rangle\left\|_{2} /\right\||\psi\rangle \|_{2} \equiv \sin (\theta)$ for $0 \leq \theta \leq \frac{\pi}{4}$.

We now introduce Matrix Product States (MPS) 3, 10, 11]. Consider a chain of $N d$-level systems with the corresponding Hilbert space

$$
\mathcal{H}_{N}:=\left(\mathbb{C}^{d}\right)^{\otimes N} .
$$

We call $\left|\phi_{D}\right\rangle \in \mathcal{H}_{N}$ a Matrix Product State (MPS) with bond dimension $D$ (or, briefly, a $D$-MPS) if it can be written as

$$
\left|\phi_{D}\right\rangle=\sum_{i_{1}, \ldots, i_{N}=1}^{d} A_{i_{1}}^{[1]} A_{i_{2}}^{[2]} \cdots A_{i_{N}}^{[N]}\left|i_{1}, i_{2}, \ldots, i_{N}\right\rangle
$$

with $A_{i}^{[k]} D \times D$ matrices for $2 \leq k \leq N-1$, and $A_{i}^{[1]}$ and $A_{i}^{[N]}$ row and column vectors of length $D$, respectively [18].

Given a family $\left(\left|\psi_{N}\right\rangle\right) \equiv\left(\left|\psi_{N}\right\rangle\right)_{N \in \mathcal{N} \subset \mathbb{N}}$ of states, $\left|\psi_{N}\right\rangle \in \mathcal{H}_{N}$, we say that it can be approximated efficiently by MPS if for every $\delta>0$, there exists a sequence $\left|\phi_{N, D}\right\rangle$ of MPS with $D \equiv D(N)=O\left(\operatorname{poly}_{\delta}(N)\right)$ such that $\left\|\psi_{N}-\phi_{N, D}\right\|_{\operatorname{tr}} \leq \delta$. On the contrary, if there is some $\delta>0$ such that no sequence of MPS with polynomial bond dimension can approximate $|\psi\rangle$ up to $\delta$, we say that $\left(\left|\psi_{N}\right\rangle\right)$ cannot be approximated efficiently by $M P S$. For brevity, we will sometimes drop the word "efficiently".

We will measure entropies using the Rényi entropies

$$
S_{\alpha}(\rho)=\frac{\log \operatorname{tr} \rho^{\alpha}}{1-\alpha}, 0 \leq \alpha \leq \infty,
$$

which are a generalization of the von Neumann entropy $S(\rho)=-\operatorname{tr}[\rho \log \rho]$. In particular, $\lim _{\alpha \rightarrow 1} S_{\alpha}(\rho)=S(\rho)$. Note that all $\operatorname{logs}$ are to the basis 2 .

We aim to relate approximability by MPS to the scaling of block entropies. To this end, we first show that the error made in approximating some state by a $D$ MPS is determined by the error made when truncating the Schmidt spectrum of its bipartitions after $D$ values. Therefore, let $|\psi\rangle \in \mathcal{H}_{N}, \rho_{k}=\operatorname{tr}_{k+1, \ldots, N}|\psi\rangle\langle\psi|$, and let $\lambda_{1}^{[k]} \geq \lambda_{2}^{[k]} \geq \cdots \geq \lambda_{d^{k}}^{[k]}$ be the ordered spectrum of $\rho_{k}$. Then, define the truncation error

$$
\epsilon_{k}(D):=\sum_{i=D+1}^{k^{d}} \lambda_{i}^{[k]} .
$$

Let us now relate the truncation error to approximability by MPS. The intuition is that the best an MPS with bond dimension $D$ (i.e., Schmidt rank $D$ in any bipartition) can do is to preserve the $D$ largest eigenvalues, resulting in an error of $\epsilon_{k}(D)$ for the cut at $k$ (which can, but need not, accumulate). On the one side, it has been shown in [5] that for a state $|\psi\rangle \in \mathcal{H}_{N}$, there always exists an MPS $\left|\phi_{D}\right\rangle$ with bond dimension $D$ such that

$$
\||\psi\rangle-\left|\phi_{D}\right\rangle \|_{2} \leq 2 \sum_{k=1}^{N-1} \epsilon_{k}(D) .
$$

On the other hand, any $D$-MPS $\left|\phi_{D}\right\rangle$ satisfies

$$
\left\|\psi-\phi_{D}\right\|_{\text {tr }} \geq \epsilon_{k}(D) \quad \forall k
$$

since with $\rho_{k}=\operatorname{tr}_{1, \ldots, k} \psi$ and $\sigma_{D, k}=\operatorname{tr}_{1, \ldots, k} \phi_{D}$,

$$
\left\|\psi-\phi_{D}\right\|_{\text {tr }} \geq\left\|\rho_{k}-\sigma_{D, k}\right\|_{\text {tr }} \geq \epsilon_{k}(D) .
$$

Here, we have used: i) the contractivity of the partial trace, ii) for fixed spectra, the trace norm distance is extremal for commuting operators [12], and iii) $\operatorname{rank} \sigma_{D, k} \leq D$.

We start the discussion of Table $\llbracket$ by proving the cases for which conclusive statements can be made. In the following, $\rho_{N}^{L}$ will denote any $L$-particle reduced block of a state $\left|\psi_{N}\right\rangle \in \mathcal{H}_{N}$. The case of at most logarithmically growing Rényi entropy with $\alpha<1$ was discussed in [5], where is was shown that it implies approximability. More formally, if for a family of states $\left(\left|\psi_{N}\right\rangle\right)$ there exist $c, c^{\prime}>$ 0 and $0 \leq \alpha<1$ such that $S_{\alpha}\left(\rho_{N}^{L}\right) \leq c \log (N)+c^{\prime}$ for all reduced blocks $\rho_{N}^{L}$, then it can be approximated efficiently by MPS.

Let us now show that a linearly growing von Neumann entropy implies inapproximability. Formally, if for a family $\left(\left|\psi_{N}\right\rangle\right), S\left(\rho_{N}^{L}\right) \geq c L$ holds for some $c>0$, $L \equiv L(N) \geq \eta N, \eta>0$, and some reduced blocks $\rho_{N}^{L}$, then it cannot be approximated efficiently by MPS.

To prove this, we use Fannes' inequality in its improved version by Audenaert [6]: For density operators $\rho, \sigma$ on a $K$-dimensional Hilbert space, $|S(\rho)-S(\sigma)| \leq$ $T \log (K-1)+H(T, 1-T)$, where $2 T=\|\rho-\sigma\|_{\text {tr }} \leq \delta$, and $H(T, 1-T) \leq 1$ is the binary entropy. Let $\left(\left|\phi_{N, D}\right\rangle\right)$ be a sequence of MPS approximating $\left(\left|\psi_{N}\right\rangle\right)$, and $\rho_{N}^{L}, \sigma_{N, D}^{L}$ 
the corresponding reduces states for which $S\left(\rho_{N}^{L}\right) \geq c L$. Then,

$$
\left|S\left(\rho_{N}^{L}\right)-S\left(\sigma_{N, D}^{L}\right)\right| \leq \frac{1}{2} \delta L \log d+1,
$$

and thus, for $L \geq \eta N$,

$$
\begin{aligned}
\log D(N) \geq S\left(\sigma_{N, D}^{L}\right) & \geq S\left(\rho_{N}^{L}\right)-\frac{1}{2} \delta L \log d-1 \\
& \geq \eta\left(c-\frac{1}{2} \delta \log d\right) N-1,
\end{aligned}
$$

i.e., the bond dimension grows exponentially in $N$ as soon as the error $\delta<2 c / \log d$, which completes the proof.

In the following, we show that a faster than logarithmic increase of any Rényi entropy with $\alpha>1$ also implies inapproximability, i.e., if for a family $\left(\left|\psi_{N}\right\rangle\right)$, there exist $\alpha>1$ and $\kappa>0$ s.th. $S_{\alpha}\left(\rho_{N}^{L}\right) \geq c L^{\kappa}$ for some $c>0$, $L \equiv L(N) \geq \eta N$, and some reduced blocks $\rho_{N}^{L}$, then it cannot be approximated efficiently by MPS.

This is proven by lower bounding the truncation error $\epsilon \equiv \epsilon(D)$ of a block $\rho_{N}^{L}$ for given $S_{\alpha}(\rho)(\alpha>1)$ and then applying (4). This, however, is the same as maximizing the entropy while keeping $\epsilon$ fixed. Since both the entropy and $\epsilon$ only depend on the spectrum, the problem reduces to a classical one. It is easy to see that the probability distribution

$$
p_{1}, \ldots, p_{D}=\frac{1-\epsilon}{D} ; p_{D+1}, \ldots, p_{2^{L}}=\frac{\epsilon}{2^{L}-D}
$$

is majorized by all ordered probability distributions $\left(q_{i}\right)$ which satisfy $q_{D+1}+\cdots+q_{2^{N}}=\epsilon$, and since Rényi entropies are Schur concave functions, it has maximal entropy [12]. Therefore, we obtain the inequality

$$
\begin{aligned}
& S_{\alpha}\left(\rho_{N}^{L}\right) \leq \frac{-1}{\alpha-1} \log \left[\frac{(1-\epsilon)^{\alpha}}{D^{\alpha-1}}+\frac{\epsilon^{\alpha}}{\left(2^{L}-D\right)^{\alpha-1}}\right] \\
& \quad \leq \frac{-1}{\alpha-1} \log \left[\frac{(1-\epsilon)^{\alpha}}{D^{\alpha-1}}\right]=\log D-\frac{\alpha}{\alpha-1} \log (1-\epsilon) .
\end{aligned}
$$

Since from (41) the total error is $\delta \geq \epsilon$, we find

$$
\log D \geq S_{\alpha}\left(\rho_{N}^{L}\right)+\frac{\alpha}{\alpha-1}|\log (1-\delta)|,
$$

and from $S_{\alpha}\left(\rho_{N}^{L}\right) \geq c L^{\kappa} \geq c \eta^{\kappa} N^{\kappa}$, we infer that $D$ has to grow exponentially for any $\delta$.

We now turn towards the undetermined region in Table I. where we provide examples for both approximability and inapproximability. This task is greatly simplified by the fact that approximability examples extend to the top and left in Table I, while inapproximability extends to the right and bottom. This holds as approximability for a given scaling implies the same for more moderate scalings (and conversely for inapproximability), and since $S_{\alpha}(\rho)$ decreases monotonically in $\alpha$.

The aim of this work is to clarify the relation between entropy scaling laws and the approximability by MPS: Therefore, our examples are not constructed to be ground states. Yet, all of them form uniform families of states, i.e., they can be generated by a uniform family of time dependent Hamiltonians. The existence of time-independent realizations is plausible, as the central ingredient of the examples are properly distributed entangled pairs. These could be represented by pairs of localized excitations which are prepared locally and then propagated by a time-independent Hamiltonian.

All of the examples can be chosen to be translational invariant, with the only possible exception of the inapproximability example for constant von Neumann entropy. The question whether any translational invariant state with bounded von Neumann entropy can be approximated efficiently by MPS thus remains open.

The examples can be grouped into two classes; the first is based on states of the type

$$
\left|\psi_{2 N}\right\rangle=\sqrt{1-p_{N}}|2\rangle^{\otimes 2 N}+\sqrt{\frac{p_{N}}{2^{N}}} \sum_{x \in\{0,1\}^{N}}|x\rangle|x\rangle .
$$

By choosing $p_{N}=1 / N$, we obtain an example of a state with linearly growing Rényi entropies for all $\alpha<1$ which can be approximated by MPS, as

$$
\|\left|\psi_{2 N}\right\rangle-\sqrt{1-p_{N}}|2\rangle^{\otimes 2 N} \|_{2}=\sqrt{p_{N}} \rightarrow 0 .
$$

On the other hand, for $L \leq N$,

$$
\rho_{2 N}^{L}=\left(1-p_{N}\right)|2\rangle\left\langle\left. 2\right|^{\otimes L}+\frac{p_{N}}{2^{L}} \sum_{y \in\{0,1\}^{L}} \mid y\right\rangle\langle y|,
$$

and therefore

$$
\begin{aligned}
S_{\alpha}\left(\rho_{2 N}^{L}\right) & =\frac{1}{1-\alpha} \log \left[\left(1-p_{N}\right)^{\alpha}+2^{(1-\alpha) L} p_{N}^{\alpha}\right] \\
& \geq L-\frac{\alpha}{1-\alpha} \log N .
\end{aligned}
$$

Note that the infavourable scaling of $c_{\alpha}:=\frac{\alpha}{1-\alpha}$ for $\alpha \rightarrow 1$ can be compensated by e.g. choosing $p_{N}=N^{-1 / c_{\alpha}}$.

The next example provides states with algebraically (but sublinearly) growing von Neumann entropy which can be approximated efficiently by MPS. Therefore, fix $0<\kappa<1$ and $\epsilon>0$, and set $p_{N}=N^{-\epsilon(1-\kappa)}$ in (6). As in the previous example, $p_{N} \rightarrow 0$ implies approximability, and

$$
S\left(\rho_{2 N}^{L}\right)=H\left(p_{N}, 1-p_{N}\right)+p_{N} \log \left[2^{L}\right] \geq L / N^{\epsilon(1-\kappa)},
$$

which implies $S\left(\rho_{2 N}^{L}\right) \geq L^{\kappa}$ for $L \geq N^{\epsilon}$.

We now construct a state which obeys a strict area law for the von Neumann entropy but yet cannot be approximated by MPS. Therefore, set $M=2 N^{3}$ and define $\left|\chi_{M}\right\rangle=\left|\psi_{2 N}\right\rangle^{\otimes N^{2}}$ with $\left|\psi_{2 N}\right\rangle$ from (6), where $p_{N}=1 / N$. Then, $S\left(\rho_{M}^{L}\right)$ is at most twice the maximum entropy of a cut through $\left|\psi_{2 N}\right\rangle$, and thus

$$
S\left(\rho_{M}^{L}\right) \leq 2\left(H\left(p_{N}, 1-p_{N}\right)+p_{N} N\right) \leq 4 .
$$


To prove hardness of approximation, observe that for a given $D$, the best $D$-MPS approximation to $\left|\psi_{2 N}\right\rangle^{\otimes N^{2}}$ also carries this product structure, $\left|\phi_{D}\right\rangle^{\otimes N^{2}}$ [19]. From the multiplicativity of the fidelity and the relations following Eq. (1) one infers $T\left(\phi^{\otimes K}, \psi^{\otimes K}\right) \geq \sqrt{K / 8} T(\phi, \psi)$ for $T(\phi, \psi)^{2} \leq 2 / K$. Second, from the truncation error $\epsilon_{N}(D)$ for $\left|\psi_{2 N}\right\rangle, T\left(\phi_{D}, \psi_{2 N}\right) \geq\left(2^{N}-(D-1)\right) p_{N} / 2^{N}$ for any $D$-MPS $\left|\phi_{D}\right\rangle$. Together, this shows that $D \geq$ $2^{N}\left(1-8 T\left(\Phi_{D}, \chi_{M}\right)\right)+1$ which is exponential in the system size $M=2 N^{3}$.

It is unclear how to make this example translational invariant. However, for the adjacent cases in Table I. those examples exist: For $S \sim \log L$, take the preceding example and make it translational invariant by adding a tagging system $|10 \ldots 0\rangle^{\otimes N^{2}}$ and superposing all translations. The resulting state is hard to approximate as the translational invariance can be broken by local projections on the tags, and since the reduced state $\rho_{N}^{L}$ is the translational invariant mixture of the original, tagged reduced states, the entropy is increased by at most $\log L$. For the case $S_{\alpha} \sim$ const., $\alpha>1$, the state (6) with constant $p_{N}$ does the job.

The last two examples are of a different type: We consider $N$ spins on an ring and equidistantly distribute $\nu$ maximally entangled pairs between opposite sites (i.e., $k$ and $k+N / 2$ ), while initializing all remaining qubits to $|0\rangle$. The first example, obtained for $\nu=\log N$, provides a state with $S_{\infty} \sim \log L$ which is approximable. It is clearly an MPS with $D=2^{\log N}=N$, and for any $c>0, S_{\infty}\left(\rho_{N}^{L}\right) \geq\left\lfloor\frac{L}{N} \log N\right\rfloor \geq c \log L-1$ for $L \geq c N$. It can be made translational invariant by superposing all translates of the state: On the one hand, this increases the bond dimension by at most a factor of $N$ [11], while on the other hand, the largest eigenvalue of a block of length $N / \log N$ is $\frac{1}{2}$, i.e., the $\log L$ lower bound on the $S_{\infty}$ entropy remains unchanged.

The second example illustrates that for any $\kappa>0$, there is a state with $S_{0} \sim N^{\kappa}$ which cannot be approximated by MPS. Therefore, choose $\nu=N^{\kappa}$ : Then, $S_{0}\left(\rho_{N}^{L}\right) \leq N^{\kappa} L / N+1 \leq 2 L^{\kappa}$, while inapproximability follows from the superlogarithmic number of maximally entangled pairs. Translational invariance is achieved by taking the superposition of all translations for $\kappa^{\prime}<\kappa$. The spectrum of a block of length $N / N^{\kappa^{\prime}}$ is broadened to $\left(\frac{1}{2}, \frac{N^{\kappa^{\prime}}}{2 N}, \ldots, \frac{N^{\kappa^{\prime}}}{2 N}\right)$ : this clearly increases the truncation error, and the entropy scaling gets a log correction $S_{0}\left(\rho_{N}^{L}\right) \leq 2 L^{\kappa^{\prime}}\left(1+\left(1-\kappa^{\prime}\right) \log L\right)$ which is bounded by $4 L^{\kappa}$ for properly chosen $\kappa^{\prime}$ and $L$.

Let us now prove the hardness of simulating time evolutions with MPS-based approaches, using the results obtained (cf. also [13]). To this end, take a spin chain with all spins up, and apply a critical Ising Hamiltonian with periodic boundary conditions. There is good evidence [14] that in this case the block entropy of any block grows linearly in time, and indeed, a lower bound
$S\left(\rho_{N}^{L}(t)\right) \geq 4 t / 3 \pi+O(\log t)$ for $t \leq e L / 4$ can be rigorously proven [15]. By plugging this into (5) and setting $L=4 t / e$, one finds that for an error $\delta<2 e / 3 \pi \approx 0.58$, the required bond dimension, and thus the effort to simulate the time evolution using MPS, grows exponentially in time.

In this work, we have explored the relation between the scaling of block entropies and approximability by MPS. More refined criteria might be obtained by considering more involved figures of merit. For instance, the approximability proof of [5] can be adapted to smooth Rényi entropies $S_{\alpha}^{\epsilon}(\rho)=\min \left\{S_{\alpha}(\sigma):\|\rho-\sigma\|_{\operatorname{tr}} \leq \epsilon\right\}$ [16]. Then, the existence of $\alpha<1, \epsilon>0$, and $c>0$ s.th. $S_{\alpha}^{1 / N^{1+\epsilon}}\left(\rho_{N}^{L}\right) \leq c \log N$ implies approximability [20]. Indeed, the state (6) with $p_{N}=1 / N^{2}$ has linearly growing Rényi entropies, while the smooth Rényi entropies are constant and thus imply approximability.

We thank K. Audenaert and K. G. Vollbrecht for helpful discussions. This work was supported by the EU, the DFG, and the Elite Network of Bavaria project QCCC.

[1] S. R. White, Phys. Rev. Lett. 69, 2863 (1992).

[2] U. Schollwöck, Rev. Mod. Phys. 77, 259 (2005), condmat/0409292.

[3] G. Vidal, Phys. Rev. Lett. 93, 040502 (2004), quant$\mathrm{ph} / 0310089$.

[4] M. B. Hastings, Phys. Rev. B 76, 035114 (2007), cond-mat/0701055.

[5] F. Verstraete and J. I. Cirac, Phys. Rev. B 73, 094423 (2006), cond-mat/0505140.

[6] K. M. R. Audenaert J. Phys. A 40, 8127 (2007), quant$\mathrm{ph} / 0610146$.

[7] S. Lloyd, Science 273, 1073 (1996).

[8] M. B. Hastings, J. Stat. Mech. P08024 (2007), arXiv:0705.2024

[9] K. G. Vollbrecht and I. Cirac, Phys. Rev. A 73, 012324 (2006), quant-ph/0502143; R. Raussendorf, Phys. Rev. A 72, 052301 (2005), quant-ph/0505122, K. G. Vollbrecht and I. Cirac, Phys. Rev. Lett. 100, 010501 (2008), arXiv:0704.3432 A. Kay, Phys. Rev. A 76, 030307(R) (2007), arXiv:0704.3142

[10] A. Klümper, A. Schadschneider, and J. Zittartz, J. Phys. A 24, L955 (1991); Z. Phys. B 87, 281 (1992).

[11] D. Perez-Garcia, F. Verstraete, M. M. Wolf, and J. I. Cirac, Quant. Inf. Comput. 7, 401 (2007), quant$\mathrm{ph} / 0608197$.

[12] R. Bhatia, Matrix Analysis (Springer, New York, 1996).

[13] A. Datta and G. Vidal, Phys. Rev. A 75, 042310 (2007), quant-ph/0611157.

[14] P. Calabrese and J. Cardy, J. Stat. Mech. P04010 (2005), cond-mat/0503393.

[15] N. Schuch, M. M. Wolf, K. G. Vollbrecht, and J. I. Cirac (2008), arXiv:0801.2078

[16] R. Renner (2005), quant-ph/0512258.

[17] For extensive observables, where $\left\|\psi-\psi_{D}\right\|_{\text {tr }} \leq \delta / N$, the results are the same except that $S \sim N^{\kappa}$ now implies inapproximability [replace $\delta$ by $\delta / N$ in (5)]. In the ap- 
proximability example for linearly growing $\alpha<1$ Rényi entropy, one has to set $p_{N}=1 / N^{3}$. As an example of an inapproximable state with bounded von Neumann entropy, a single copy of (6) is now sufficient, yielding a translation invariant example.

[18] Using MPS with periodic boundary conditions (PBC) gives the same results, as any PBC MPS with bond dimension $D$ can be embedded in an MPS with open boundaries (2) with bond dimension $D^{2}$.

[19] We prove that the optimal $D$-MPS approximating $\left|\psi_{A}\right\rangle\left|\psi_{B}\right\rangle \in \mathcal{H}_{K} \otimes \mathcal{H}_{L}(K+L=N)$ can always be chosen to carry the same product structure: Given a $D$-MPS $\left|\phi_{D}\right\rangle$ as in (2), write it as $\left|\phi_{D}\right\rangle=\sum_{k}\left|\alpha_{k}\right\rangle\left|\beta_{k}\right\rangle$, with

$$
\begin{aligned}
\left|\alpha_{k}\right\rangle & =\sum A_{i_{1}}^{[1]} \cdots A_{i_{K}}^{[K]} e_{k}\left|i_{1}, \ldots, i_{K}\right\rangle, \\
\left|\beta_{k}\right\rangle & =\sum e_{k}^{\dagger} A_{i_{K+1}}^{[K+1]} \cdots A_{i_{N}}^{[N]}\left|i_{1}, \ldots, i_{N}\right\rangle,
\end{aligned}
$$

where $e_{k}$ is the $k$ 'th unit vector. Since the $\left|\alpha_{k}\right\rangle\left(\left|\beta_{k}\right\rangle\right)$ differ only by one boundary condition, any superposition thereof, and in particular the orthonormal vectors $\left|\tilde{\alpha}_{k}\right\rangle,\left|\tilde{\beta}_{k}\right\rangle$ appearing in the Schmidt decomposition $\left|\phi_{D}\right\rangle=\sum_{k} \lambda_{k}\left|\tilde{\alpha}_{k}\right\rangle\left|\tilde{\beta}_{k}\right\rangle$, are D-MPS. Define $a_{k}:=$ $\left\langle\psi_{A} \mid \tilde{\alpha}_{k}\right\rangle, b_{k}:=\left\langle\psi_{B} \mid \tilde{\beta}_{k}\right\rangle$, and the factorizing $D$-MPS

$$
\left|\phi_{D}^{\prime}\right\rangle=\left(\sum_{k} \lambda_{k} \frac{\left|a_{k}\right|}{a_{k}}\left|\tilde{\alpha}_{k}\right\rangle\right)\left(\frac{1}{|\mathcal{L}|^{1 / 2}} \sum_{l \in \mathcal{L}} \frac{\left|b_{l}\right|}{b_{l}}\left|\tilde{\beta}_{l}\right\rangle\right),
$$

where $\mathcal{L}=\left\{l:\left|b_{l}\right| \geq\left|b_{j}\right| \forall j\right\}$. Then, $\left|\phi_{D}^{\prime}\right\rangle$ is a normalized $D$-MPS, and $\left|\left\langle\psi_{A}, \psi_{B} \mid \phi_{D}\right\rangle\right|<\left|\left\langle\psi_{A}, \psi_{B} \mid \phi_{D}^{\prime}\right\rangle\right|$ unless the Schmidt rank of $\left|\phi_{D}\right\rangle$ is one.

[20] This is tight: The scaling, as smooth entropies are lower bounds on their non-smooth version, and the smoothening, as the inapproximability example for constant von Neumann entropy also has constant $S_{\alpha}^{5 / N}$ entropy. Conversely, for every approximable state, $S_{\alpha}^{\epsilon}\left(\rho_{N}^{L}\right)$ grows at most logarithmically for every $\epsilon$. This is also tightconsider, e.g., (6) with $p_{N}=1 / N^{\kappa}$. 